\title{
Obstacles to Implementing the HPV Vaccine: Is it Worth Pursuing or Not?
}

\author{
Mastura Mohd Sopian1, Sharifah Azdiana Tuan Din², Hasmah Hussin²
}

${ }^{1}$ Oncological radiological Sciences Cluster, Advanced Medical and Dental Institute, Universiti Sains Malaysia, 13200, Kepala Batas, Pulau Pinang, Malaysia. ${ }^{2}$ Regenerative Medicine Cluster Advanced Medical and Dental Institute, Universiti Sains Malaysia, 13200, Kepala Batas, Pulau Pinang, Malaysia.

\begin{abstract}
Cervical cancer, along with breast, colorectal, and lung cancer, has become increasingly problematic in Malaysia. The prevalence of cervical cancer in Malaysia is projected to rise in younger women, and Malaysia views this prediction with concern and awareness of the need to take action to prevent the illness among those who are currently healthy. Providing free HPV vaccination is a way to lower the risk of developing cervical cancer among women in Malaysia. However, while Malaysia has been able to provide this vaccination at minimal or no cost, changes in socio-economic circumstances have stretched the demand. This review aims to highlight the probability of HPV vaccination acceptance and the reasons for it. An extensive literature review of acceptance, knowledge, attitude, practice, and decision making about HPV vaccination was performed to describe issues related to vaccination. The evidence presented herein can help identify ways to improve the HPV vaccination program in Malaysia.
\end{abstract}

Keywords: Papillomavirus- vaccines- barriers- acceptance

Asian Pac J Cancer Care, 4 (4), 165-169

\section{Introduction}

Worldwide, cervical cancer is the fourth most common cancer in women. In Malaysia, cervical cancer is ranked second among all cancers in women aged 15 to 44 years after breast cancer. In Malaysia, women are at risk for cervical cancer as early as age 15 , and approximately 2145 cases of cervical cancer were diagnosed in 2012. Cervical cancer is the fifth leading cause of cancer deaths in women, and 621 new cervical cancer deaths occur annually in Malaysia. The incidence of cervical cancer in younger women rose dramatically during the decades [1]. This trend is a worrisome, as it affects future generations and it occurs during the prime productive years. Cervical cancer is an important public health concern because of the high burden of the disease and the potential for preventing it via an effective screening program [2]. Pap smears have proved to be an important screening method, as nationwide screening resulted in the successful reduction of cervical cancer incidence up to $80 \%$ [3]. However, despite awareness campaigns conducted by government and non-
Submission Date: 04/07/2019 Acceptance Date: 07/24/2019

government agencies, Pap smear screening performance in Malaysia remains low [4]. Cervical cancer is caused by human papillomavirus (HPV) [1-5-6]. Vaginal bleeding after sexual intercourse and bleeding between menstrual periods are among the symptoms of cervical cancer. Unfortunately, however, there often are no obvious signs or symptoms during the early stages of the disease. The majority of cases are squamous cell carcinoma followed by adenocarcinoma [6].

HPV also is strongly related to anogenital and head and neck cancer. Increasingly, evidence strongly links HPV DNA with cancers of the cervix, anus, vulva, vagina, and penis. However, cancers of the anus, vulva, vagina, and penis are less frequent compared to cervical cancer. Nevertheless, their association with HPV means that they potentially are preventable with HPV vaccination [1].

\section{Risk factors for cervical cancer}

In Malaysia, the most frequent HPV oncogenic types among women with invasive cervical cancer as determined by histology are HPV 16 and HPV 18 [6]. Four major steps

\footnotetext{
Corresponding Author:

Dr. Mastura Mohd Sopian

Oncological radiological Sciences Cluster, Advanced Medical and Dental Institute, Universiti Sains Malaysia, 13200, Kepala Batas, Pulau

Pinang, Malaysia.

Email: mastura_sopian@usm.my
} 
are involved in the development of cervical cancer: HPV infection, viral persistence, progression to precancerous lesions, and invasion through the basement membrane of the epithelium [1]. Persistent infections and precancerous lesions are established within 5-10 years in $<10 \%$ of new infections. Thus, invasive cancer may develop in women with precancerous lesions over many years [1-4-7].

Sexual intercourse is the primary route of transmission of genital HPV infection. Therefore, early onset of sexual activity ( $<16$ years old), more than four sexual partners, and history of genital warts are associated with a high risk of cervical cancer [1-8]. Male circumcision and the use of condoms have been shown to have a significant protective effect against HPV transmission [1-5]. Other cofactors that contribute to the progression of cervical cancer are smoking, high parity, long-term hormonal contraceptive use, and co-infection with HIV. Co-infection with chlamydia and herpes simplex virus type- 2 and immunosuppression are other probable cofactors [1].

\section{HPV preventive strategies}

The Pap smear test is an established and well-organized cervical screening program that began in Malaysia in the 1960s [9], and it is available at all government health facilities and hospitals as well as in private settings [5]. Such screening is needed to limit the country's burden of cervical cancer, and other preventive strategies, such as HPV vaccination, should be implemented.

In 2007, Universiti Kebangsaan Malaysia conducted a study of the cost-effectiveness of HPV vaccination for the prevention of cervical cancer in Malaysia. The findings showed that the government spends RM382 million for cervical cancer treatment annually, whereas providing the vaccine to prevent cervical cancer cost only RM150 million a year [10]. Thus, vaccination of young women is a cost-effective strategy to combat cervical cancer.

In 2006, the US Food and Drug Administration (FDA) approved the prophylactic use of the HPV vaccine, and it is recommended that women aged 9 to 26 years should be vaccinated (MMWR Morbidity and mortality weekly report, 2010). The vaccines are made from non-infectious HPV virus-like particles (VLPs). The two types of HPV vaccine licensed by the FDA are the bivalent vaccine (Cervarix) and the quadrivalent vaccine (Gardasil). Cervarix protects against HPV 16 HPV 18, which cause $70 \%$ of cervical cancers. Gardasil protects against HPV types $6,11,16$, and 18 , of which types 6 and 11 cause $90 \%$ of genital warts cases. Only the quadrivalent vaccine is used for males. Neither of the vaccines has therapeutic effects on HPV-related disease (MMWR Morbidity and mortality weekly report, 2010). However, both vaccines have shown excellent efficacy and safety (MMWR Morbidity and mortality weekly report, 2010). The quadrivalent HPV vaccine is routinely recommended, as it is $90.7-100 \%$ effective in preventing infection of the four HPV types, and it also is safe and halal [11]. However, recommendations regarding HPV vaccination vary among countries.

\section{Prevalence of Pap smear testing}

Although the Pap smear has been available in Malaysia since the 1960s, no reduction in cervical cancer prevalence has been documented [9]. The National Health and Morbidity Survey revealed that the national prevalence of Pap smear testing was $43.7 \%$, and only $59.7 \%$ were performed within the last 3 years [4-12], reported that in 2000 and 2006, among cervical cancer patients who were diagnosed in major hospitals in Malaysia, 48\% reported never having had a Pap smear and $95 \%$ had not had a Pap smear within the past 3 years.

The Ministry of Health Malaysia recommends that all women aged 20 to 65 years who are or who have been sexually active undergo Pap smear testing. If two consecutive yearly tests are negative, screening every 3 years is recommended [5]. The American Cancer Society and the U.S. Preventive Services Task Force recommend Pap smear screening in women age 21 to 65 years every 3 years [13-14]. Although there is great variation among countries, the overall lowest cervical cancer screening rate in the U.S. is among Asian women [15].

\section{Prevalence of HPV vaccine acceptance}

A cross-sectional study among mothers conducted in 2007 showed that $65.7 \%$ of respondents accepted HPV vaccination for their daughters. However, $83.9 \%$ of the mothers were unwilling to vaccinate their children if they had to pay, but the acceptability rate increased to $97.8 \%$ if it was routine and freely available [16]. Generally, the rate of HPV vaccination has been low when the vaccination program is individually funded and voluntarily. Compared to the U.S. and Europe, the rate of HPV vaccination has been low in Asian countries [17-18]. Studies conducted in Hong Kong, Korea, Taiwan, India, China, Thailand, and Malaysia reported very low rates $(\sim 20.9 \%)$ of HPV vaccination [18-19].

A national survey conducted in China found that mothers were less likely to accept the HPV vaccine [20]. Studies conducted in Thailand and China reported rates of $40.9 \%$ and $36.2 \%$, respectively [17-20]. Higher acceptance rates were also found in India and Indonesia [21-24]. reported high acceptability in the UK, as $75-81 \%$ of parents said they would like to vaccinate their daughters.

It is reassuring that the greatest contributing factor to the high acceptability of HPV vaccination in Malaysia is the endorsement of the HPV vaccine as part of the national immunization program [25]. The ideal time for vaccination (i.e., to provide the greatest protection) is before a person becomes sexually active. Epidemiological studies in Malaysia have revealed cases of cervical cancer in women as young as 15 years old [1]. Due to the young age at which vaccination is needed, it must be given with parental consent. Therefore, the success of the HPV vaccination program depends on the parents. This means that explanations of the rationale for HPV vaccination age selection and information about the sexual behaviors of young adolescents should be incorporated into educational materials for parents. 
Barriers to HPV vaccination

Major obstacles to the acceptability of HPV vaccination include high cost and fear of side effects, multiple injections, and social stigma [26]. The cost of the HPV vaccine was a serious barrier to the success of the vaccination program in Malaysia before 2010 [26]. Parents also may fear that vaccination might promote sexuality and result in increased unsafe sexual behavior [16-17-21]. Additionally, due to religious and moral values, some parents may believe that their children are at low risk for contracting the infection and that vaccination is, therefore, unnecessary [21]. Some parents also are concerned about the efficacy and safety of the vaccines [26]. Moreover, many parents who refuse HPV vaccination for their children do so because of concern about halal and efficacy.

In one study, the major concerns for those who opposed the HPV vaccine were the newness of the vaccine and its safety and efficacy, and this was especially true for the fathers [27]. One of the most common reasons that fathers did not agree to vaccinate their daughters was worry about possible side effects (64.5\%) [28-29] reported that the most common reasons for not vaccinating daughters in Singapore were inadequate information and unproven vaccine effectiveness. Similarly, the most influential factors impacting HPV vaccination in China and the Philippines were worries about the efficacy and safety of the vaccine [30-31]. In a 2007 study about acceptance of HPV vaccination, the halal issue of the HPV vaccine was an important factor affecting the acceptance of HPV vaccine among Muslim mothers [3].

Sexual issues are taboo in many Asian cultures, and parents may find it difficult to explain to their children the need for vaccination against sexually transmitted infections [26]. In one study conducted in India, parents believed that their daughters were unlikely to become sexually active before marriage [21]. These issues may explain why HPV vaccination was not accepted among parents in those studies. However, most parents also believed that the vaccine would not promote risky sexual behaviors among teenagers which were similar to results reported by [16-17]. Thus, this may explain the high acceptance rate in their studies. In contrast, a study conducted in the UK noted that the principle of adolescent autonomy is recognized there, and it might contribute to the view that the vaccine encourages sexual promiscuity [24-32] reported that teenage pregnancy and consistent condom use have been associated with adolescents' intent to receive the HPV vaccine. This finding may be due to worry among parents about the age for vaccination against sexually transmitted infections and to adolescents' misconceptions. A study in an Islamic society in Turkey reported that most participants $(95 \%)$ disagreed that being vaccinated had the potential to encourage early sexual activity or promiscuity [33]. In Asian culture, however, communication about sexuality at home and in the school environment is not as open as it is in European countries.

To address concerns about increased sexual behavior among adolescents, communication within families about religious and sexual health should be emphasized.
With such communication, parents can make clear that adolescents should not interpret parental approval of HPV vaccination as condoning early sexual behavior [34].

To date, many studies have assessed factors that influence the level of knowledge and acceptance of HPV vaccination among women from various groups in Malaysia. Several studies evaluated demographic factors associated with parental attitudes towards HPV vaccination [17-21-35]. Factors related to acceptance of the vaccine include age [16-22], gender [20-30], education level [20], employment status [20], and income (16, 20). Other studies reported that increasing age, female gender, higher education attainment, and low household income were significantly associated with increased HPV vaccine acceptance [16-26-31-36].

Studies of how knowledgeable people are about HPV infection and vaccination were conducted in various groups, including secondary school students [17-37], teachers [17], parents [17], university students [38], and other groups of women from various levels of education [39-40]. They concluded that overall knowledge about HPV infection, vaccination, and cervical cancer is poor. The general lack of knowledge about HPV is a major factor that might adversely affect HPV vaccine acceptability.

Factors that influence the HPV vaccination decision include physician recommendation, parents' and adolescents' attitudes and knowledge, social-environmental factors, institutional policies, and interventions related to the vaccine. Cultural beliefs and values of parents can affect the decision [32]. It was found that there is no difference in sex role orientation among the families, and wives with a higher level of education play a greater role in decision making. However, one study reported that husbands have a greater role in making major decisions, such as those related to health, education, and insurance [41]. Additionally, fathers appeared to be less accepting and knowledgeable about HPV vaccination than mothers [17-21-35]. In contrast to these reports, however, April et al. (2010) found that mothers were the most influential in deciding about HPV vaccination (73\%). Mothers are the primary communicators with daughters about sex-related topics such as contraception, reproduction, and physical and sexual development [32]. This likely explains why mothers are more accepting of the HPV vaccine than fathers. A study conducted in India reported that the HPV vaccine decision was most commonly made jointly decision by father and mother [21].

Because the decision to vaccinate is influenced by parental factors, it will be helpful to investigate family decision making related to general childhood vaccinations to better understand the decision of whether or not to vaccinate against HPV.

Age was another factor found to be significantly associated with HPV vaccine acceptance [16-22]. A study conducted among parents showed that vaccine acceptance is occurring more among older age groups [42]. Better exposure to information and greater awareness likely are two factors contributing to this trend. However, several other studies reported that parents of younger aged were more likely to accept HPV vaccine than parents of older 
aged [16-17-22]. This may be because HPV vaccination in Thailand, and Indonesia were individually funded and voluntarily, and younger parents would be more inclined to seek treatment due to their ability to pay service fees.

In conclusion, the HPV vaccine is proved to be an effective method to prevent cervical cancer. Acceptance of HPV vaccination varies among countries, and increasing HPV vaccine acceptance is a big challenge to both communities and policy makers. Therefore HPV vaccination should be encouraged at community and government levels. A step-wise approach is necessary for the common good. It would be advantageous to have in place a good communication process that would ensure acceptance.

\section{Conflict of interest}

The authors have no potential conflict of interest relevant to this article.

\section{References}

1. ICO HPV Information Centre. Human Papillomavirus and Related Diseases Report, Malaysia (2014). Available from http://www.hpvcentre.net/statistics/reports/MYS_FS.pdf. [

2. Ezat WP, Aljunid S. Cost-effectiveness of HPV vaccination in the prevention of cervical cancer in Malaysia. Asian Pacific journal of cancer prevention : APJCP. 2010;11(1):79-90.

3. LP W. Preventing cervical cancer through human papillomavirus vaccination: Perspective from focus groups. American Society for Colposcopy and Cervical Pathology. Journal of Lower Genital Tract Disease. 2009;13:85-93.

4. Othman NH, Devi BC, Halimah Y. Cervical cancer screening: patients understanding in major hospitals in Malaysia. Asian Pacific journal of cancer prevention : APJCP. 2009;10(4):569-74.

5. Clinical Practice Guidelines (CPG) Management of Cancer Cervix. Ministry of Health Malaysia (2003). Available from http://www.moh. gov.my/index.php/pages/view/148. [

6. Zaridah S. A review of cervical cancer research in malaysia. The Medical journal of Malaysia. 2014;69 Suppl A:33-41.

7. Wong LP, Wong YL, Low WY, Khoo EM, Shuib R. Knowledge and awareness of cervical cancer and screening among Malaysian women who have never had a Pap smear: a qualitative study. Singapore medical journal. 2009;50(1):49-53.

8. SE W. Cervical cancer. The Lancet. (2003);36:2217-25.

9. Nor Hayati O. Cancer of the cervix - from bleak past to bright future; a review, with an emphasis on cancer of the cervix in malaysia. The Malaysian journal of medical sciences : MJMS. 2003;10(1):13-26.

10. Aljunid S, Zafar A, Saperi S, Amrizal M. Burden of disease associated with cervical cancer in malaysia and potential costs and consequences of HPV vaccination. Asian Pacific journal of cancer prevention : APJCP. 2010;11(6):1551-9.

11. Kane MA, Sherris J, Coursaget P, Aguado T, Cutts F. Chapter 15: HPV vaccine use in the developing world. Vaccine. 2006;24 Suppl 3:S3/132-9.

12. (MOH) MoHM. The Third National Health and Morbidity Survey (NHMS III). Institute for Public Health (IPH), Kuala Lumpur. (2006).

13. Saslow D, Solomon D, Lawson HW, Killackey M, Kulasingam SL, Cain J, et al. American Cancer Society, American Society for Colposcopy and Cervical Pathology, and American Society for Clinical Pathology screening guidelines for the prevention and early detection of cervical cancer. CA: a cancer journal for clinicians. 2012;62(3):14772.

14. Moyer VA. Screening for cervical cancer: U.S. Preventive Services Task Force recommendation statement. Annals of internal medicine. 2012;156(12):880-91, w312.

15. McCracken M, Olsen M, Chen MS, Jr., Jemal A, Thun $\mathrm{M}$, Cokkinides V, et al. Cancer incidence, mortality, and associated risk factors among Asian Americans of Chinese, Filipino, Vietnamese, Korean, and Japanese ethnicities. CA: a cancer journal for clinicians. 2007;57(4):190-205.

16. Sam IC, Wong LP, Rampal S, Leong YH, Pang CF, Tai $\mathrm{YT}$, et al. Maternal acceptance of human papillomavirus vaccine in Malaysia. The Journal of adolescent health : official publication of the Society for Adolescent Medicine. 2009;44(6):610-2.

17. Songthap A, Pitisuttithum P, Kaewkungwal J, Fungladda W, Bussaratid V. Knowledge, attitudes, and acceptability of a human papilloma virus vaccine among students, parents and teachers in Thailand. The Southeast Asian journal of tropical medicine and public health. 2012;43(2):340-53.

18. Chow SN, Soon R, Park JS, Pancharoen C, Qiao YL, Basu $\mathrm{P}$, et al. Knowledge, attitudes, and communication around human papillomavirus (HPV) vaccination amongst urban Asian mothers and physicians. Vaccine. 2010;28(22):380917.

19. O H. Knowledge about HPV and the attitudes toward HPV vaccination among adult women in Asian Countries: A literature review. . Journal Korean Oncology Nurs. (2011). ;11:171-8.

20. Zhang SK, Pan XF, Wang SM, Yang CX, Gao XH, Wang ZZ, et al. Perceptions and acceptability of HPV vaccination among parents of young adolescents: a multicenter national survey in China. Vaccine. 2013;31(32):3244-9.

21. Madhivanan P, Krupp K, Yashodha MN, Marlow L, Klausner JD, Reingold AL. Attitudes toward HPV vaccination among parents of adolescent girls in Mysore, India. Vaccine. 2009;27(38):5203-8.

22. Jaspers L, Budiningsih S, Wolterbeek R, Henderson FC, Peters AA. Parental acceptance of human papillomavirus (HPV) vaccination in Indonesia: a cross-sectional study. Vaccine. 2011;29(44):7785-93.

23. Marlow LA, Waller J, Wardle J. Parental attitudes to prepubertal HPV vaccination. Vaccine. 2007;25(11):1945-52.

24. Brabin L, Roberts SA, Kitchener HC. A semi-qualitative study of attitudes to vaccinating adolescents against human papillomavirus without parental consent. BMC public health. 2007;7:20.

25. Saidatul NB RJ. Introduction of National HPV Immunisation Program:Sharing Malaysia's Experience. In: Health P, editor New York 2010. (2010). .

26. Wong L, Sam I. Current issues facing the introduction of human papillomavirus vaccine in malaysia. Malaysian family physician : the official journal of the Academy of Family Physicians of Malaysia. 2007;2(2):47-53.

27. Wong LP. Young multiethnic women's attitudes toward the HPV vaccine and HPV vaccination. International journal of gynaecology and obstetrics: the official organ of the International Federation of Gynaecology and Obstetrics. 2008;103(2):131-5.

28. Menmun S ES, Gulten G, Aygui A Attitudes to HPV Vaccination among Parents of Children Aged 10 to 13 Years. . Journal Pediatric Adolesc Gynecol (2015).28, :382-6.

29. Tay SK, Tesalona KC, Rashid NM, Tai EY, Najib SM. Vaccine Misconceptions and Low HPV Vaccination Take- 
up Rates in Singapore. Asian Pacific journal of cancer prevention : APJCP. 2015;16(12):5119-24.

30. Wang W, Ma Y, Wang X, Zou H, Zhao F, Wang S, et al. Acceptability of human papillomavirus vaccine among parents of junior middle school students in Jinan, China. Vaccine. 2015;33(22):2570-6.

31. Young AM, Crosby RA, Jagger KS, Richardson MB, Kloha RA, Safarian V. HPV vaccine acceptability among women in the Philippines. Asian Pacific journal of cancer prevention : APJCP. 2010;11(6):1781-7.

32. Gamble HL, Klosky JL, Parra GR, Randolph ME. Factors influencing familial decision-making regarding human papillomavirus vaccination. Journal of pediatric psychology. 2010;35(7):704-15.

33. Ilter E, Celik A, Haliloglu B, Unlugedik E, Midi A, Gunduz T, et al. Women's knowledge of Pap smear test and human papillomavirus: acceptance of HPV vaccination to themselves and their daughters in an Islamic society. International journal of gynecological cancer: official journal of the International Gynecological Cancer Society. 2010;20(6):1058-62.

34. Zimet GD. Improving adolescent health: focus on HPV vaccine acceptance. The Journal of adolescent health : official publication of the Society for Adolescent Medicine. 2005;37(6 Suppl):S17-23.

35. Dahlstrom LA, Tran TN, Lundholm C, Young C, Sundstrom K, Sparen P. Attitudes to HPV vaccination among parents of children aged 12-15 years-a population-based survey in Sweden. International journal of cancer. 2010;126(2):500-7.

36. Al-Naggar RA, Bobryshev YV, Al-Jashamy K, Al-Musli $M$. Practice of HPV vaccine and associated factors among school girls in Melaka, Malaysia. Asian Pacific journal of cancer prevention : APJCP. 2012;13(8):3835-40.

37. Rashwan H, Lubis SH, Ni KA. Knowledge of cervical cancer and acceptance of HPV vaccination among secondary school students in Sarawak, Malaysia. Asian Pacific journal of cancer prevention : APJCP. 2011;12(7):1837-41.

38. Al-Naggar RA, Al-Jashamy K, Chen R. Perceptions and opinions regarding human papilloma virus vaccination among young women in Malaysia. Asian Pacific journal of cancer prevention : APJCP. 2010;11(6):1515-21.

39. Al-Dubai SA, Alshagga MA, Al-Naggar RA, Al-Jashamy $\mathrm{K}$, Baobaid MF, Tuang CP, et al. Knowledge, attitudes and barriers for human papilloma virus (HPV) vaccines among Malaysian women. Asian Pacific journal of cancer prevention : APJCP. 2010;11(4):887-92.

40. Wong LP. Knowledge and attitudes about HPV infection, HPV vaccination, and cervical cancer among rural southeast Asian women. International journal of behavioral medicine. 2011;18(2):105-11.

41. Yusof SA DJ. Household decision-making and expenditure patterns of married men and women in Malaysia. Journal of family and economic issues. (2010);31, :371-81.

42. Mohd Sopian M, Shaaban J, Mohd Yusoff SS, Wan Mohamad WMZ. Knowledge, Decision-Making and Acceptance of Human Papilloma Virus Vaccination among Parents of Primary School Students in Kota Bharu, Kelantan, Malaysia. Asian Pacific journal of cancer prevention: APJCP. 2018;19(6):1509-14.

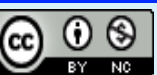

This work is licensed under a Creative Commons AttributionNon Commercial 4.0 International License. 